\title{
An Infant with Motor Delay and Deafness Due to Usher Syndrome
}

\author{
Bhanudeep Singanamalla ${ }^{1} \cdot$ Chandana Bhagwat $^{1} \cdot$ Priyanka Madaan $^{1,2} \cdot$ Gyanaranjan Nayak $^{3} \cdot$ Lokesh Saini $^{1}$ (i)
}

Received: 26 March 2021 / Accepted: 30 April 2021 / Published online: 31 May 2021

(C) Dr. K C Chaudhuri Foundation 2021

To the Editor: An 11-mo-old girl, with unremarkable perinatal and family history, presented with developmental delay, hypotonia, and hearing impairment since early infancy. Examination revealed small head, normal fundus, and central hypotonia. Clinical possibilities of previously undiagnosed kernicterus, metabolic disorders (biotinidase deficiency, mitochondrial disorders), and genetic syndromes with hearing impairment were considered. Magnetic Resonance Imaging (MRI) brain revealed nonspecific T2/FLAIR central tegmental tract hyperintensities. Metabolic workup was within normal limits. Next-generation sequencing confirmed the diagnosis of Usher syndrome (USH) with a likely pathogenic homozygous variant [c.5945G > A (p.Gly1982Arg)] in MYO7A gene. The in silico predictions of this variant are damaging by SIFT, LRT, and MutationTaster2. The observed variation has been previously reported in patients with USH [1].

USH is a rare ciliopathy, characterized by congenital sensorineural hearing loss and progressive vision loss [due to retinitis pigmentosa (RP)], usually after many years [2]. Clinical possibility of USH should be kept in a child with unexplainable developmental delay, hearing impairment with or without RP, unremarkable neuroimaging, and family history of hearing impairment or RP. In infants, the diagnosis of USH is challenging, because the clinical presentation can mimic several neurometabolic disorders and the hypotonic phase of dyskinetic cerebral palsy due to kernicterus. However, the absence of clinical signs (seen in kernicterus) such as upgaze palsy, rhythmic tongue thrusting, and brain
MRI findings can aid in thinking beyond kernicterus [3, 4]. Also, retinal pigmentary abnormalities usually take time to develop in USH, and the absence of this finding in an infantile presentation can be misleading, especially if the child has additional neurological complaints. In addition, the central tegmental tract hyperintensities seen in the index child were deceptive. Clinicians should be familiar with this finding which is nonspecific and can be seen in a variety of neurological disorders, such as cerebral palsy, epilepsy, etc.

\section{Declarations}

Conflict of Interest None.

\section{References}

1. Riazuddin S, Nazli S, Ahmed ZM, et al. Mutation spectrum of MYO7A and evaluation of a novel nonsyndromic deafness DFNB2 allele with residual function. Hum Mutat. 2008;29:502-11.

2. Tsang SH, Aycinena ARP, Sharma T. Ciliopathy: Usher syndrome. Adv Exp Med Biol. 2018;1085:167-70.

3. Madaan P, Reddy C, Saini L. Gaze Palsy: an important diagnostic clue. J Pediatr. 2019;212:236.

4. Singanamala B, Noolu R, Madaan P, Saini L. Rhythmic tongue thrusting: a useful clinical sign. Pediatr Neurol. 2020;102:81-2.

Publisher's Note Springer Nature remains neutral with regard to jurisdictional claims in published maps and institutional affiliations.

Lokesh Saini

drlokeshsaini@gmail.com

1 Pediatric Neurology Unit, Department of Pediatrics, Advanced Pediatrics Centre, Post Graduate Institute of Medical Education \& Research (PGIMER), Chandigarh 160012, India

2 Council of Scientific and Industrial Research, New Delhi, India

3 Department of Otolaryngology and Head and Neck Surgery, PGIMER, Chandigarh, India 